\title{
Deformação Residual Longitudinal de Clones de Eucalyptus em Local Sujeito a Ventos
}

\author{
Rafael Leite Braz ${ }^{1}$, José Tarcísio da Silva Oliveira² ${ }^{2}$ Antônio Marcos Rosado², \\ Graziela Baptista Vidaurre ${ }^{2}$, José Geraldo Lima de Oliveira²
}

\begin{abstract}
${ }^{1}$ Departamento de Ciência Florestal - DCFL, Universidade Federal Rural de Pernambuco - UFRPE, Recife/PE, Brasil ${ }^{2}$ Departamento de Ciências Florestais e da Madeira - DCFM, Universidade Federal do Espírito Santo - UFES, Jerônimo Monteiro/ES, Brasil

${ }^{3}$ Eldorado Brasil Celulose e Papel, Três Lagoas/MS, Brasil
\end{abstract}

\begin{abstract}
RESUMO
Durante o desenvolvimento das árvores, esforços mecânicos são gerados com o intuito de mantê-la em equilíbrio e ereta em resposta a diversos fatores ambientais. Estes esforços são conhecidos como tensões de crescimento e variam na sua distribuição e intensidade nas diferentes espécies do gênero Eucalyptus. Portanto, objetivou-se avaliar a deformação residual longitudinal (DRL) e sua relação com as características de crescimento da árvore, propriedade física e mecânica da madeira de clones do híbrido Eucalyptus urophylla $\times$ Eucalyptus grandis com 30 e 35 meses de idade, em plantios florestais na região do Vale do Rio Doce, Minas Gerais, em áreas sujeita a forte ação dos ventos, que tem como consequência o tombamento e até mesmo a quebra dessas árvores. A DRL média foi de $0,086 \mathrm{~mm}$, e quando associada as outras características da madeira, as correlações não foram significativas. As características de crescimento das árvores, a densidade básica, o módulo de elasticidade (MOE) e módulo de ruptura (MOR) e trabalho absorvido não foram suficientes para explicar o comportamento da DRL.
\end{abstract}

Palavras-chave: tensão de crescimento, vento, Eucalyptus urophylla $\times$ Eucalyptus grandis.

\section{Longitudinal Residual Strain of Eucalyptus Clone in Location Subject to Action of Winds}

\begin{abstract}
During development and growth of trees, mechanical stresses are generated in order to keep it balanced and upright in response to various environmental factors. These efforts are known as growth stresses and can vary in distribution and intensity across different species of the genus Eucalyptus. Thus, the objective was to evaluate the longitudinal residual strains (LRS) and its correlations with tree growth features, physical and mechanical properties of the Eucalyptus urophylla $\times$ Eucalyptus grandis hybrid clones. Trees were 30 and 35 months old, located in forest plantations of Vale do Rio Doce, Minas Gerais, in areas subject to wind action, with damping effect and even the breaking of these trees. The LRS measured with extensometer was on average $0.086 \mathrm{~mm}$ and when associated with other characteristics of the wood, the correlations were not significant. The growth characteristics of trees, basic density, modulus of elasticity (MOE) and modulus of rupture (MOR), and absorbed work were not enough to explain the LRS behavior.
\end{abstract}

Keywords: stress growth, wind, Eucalyptus urophylla $\times$ Eucalyptus grandis. 


\section{INTRODUÇÃO}

Conforme Lima et al. (2004), as tensões de crescimento são forças que agem sobre os tecidos das árvores, de forma a mantê-las íntegras e eretas, garantindo resistência à ruptura mecânica e à flexão no tronco contra a ação dos ventos.

As tensões de crescimento na madeira são oriundas de forças que se desenvolvem no interior dos troncos em árvores vivas (Dinwoodie, 1966). Estas tensões são características inerentes ao crescimento natural das árvores, sendo mais acentuadas em folhosas do que em coníferas (Latorraca \& Albuquerque, 2000) e contribuem com as árvores ao dar-lhes estabilidade e na manutenção do equilíbrio em resposta a agentes ambientais (Wyk, 1978; Souza, 2002).

De acordo com Opie et al. (1984), Rech \& Silva (2001a, b), há suspeita de que as causas de altas tensões de crescimento nas árvores estejam relacionadas a fatores genéticos, idades, dimensões da tora, taxa de crescimento e inclinação do fuste.

Del Menezzi (1999) ressalta que muitos fatores externos, ambientais ou silviculturais, tais como a taxa de crescimento, a desrama, o desbaste, o espaçamento, o manejo florestal, o tipo de solo e os ventos podem influenciar na intensidade das tensões de crescimento. Carvalho et al. (2010) descrevem que as limitações à utilização da madeira de eucalipto por causa da presença de tensões são mais notáveis em florestas jovens que em adultas, como consequência de diversos fatores, entre eles a proporção de lenho juvenil.

Baena (1982) relata que a intensidade de tensões de crescimento tende a ser mais elevada em indivíduos mais jovens, reduzindo consideravelmente à medida que a arvore envelhece. Para Hillis \& Brown (1978), os níveis de tensões de crescimento são maiores em épocas mais chuvosas que em seca.

O papel principal destas tensões é fornecer suporte à árvore por meio da sua ativação, pois a árvore regula a posição de sua copa em resposta às condições ambientais a que estão sujeitas. Como as árvores estão imóveis, a ação das tensões em um de seus lados permite que ela se curve (Kubler, 1987). Huang et al. (2010) relataram que essas forças existentes representam um importante mecanismo biomecânico que permite às árvores se ajustarem conforme o seu ambiente ecológico.
A seleção de material com menores níveis de tensões de crescimento e com melhor distribuição na circunferência do tronco deve ser priorizada nos programas de melhoramento genético florestal. Para isso existe a necessidade da avaliação dos níveis de tensões de crescimento na circunferência no tronco das árvores ainda vivas (Trugilho et al., 2006).

Na região do Vale do Rio Doce, no Estado de Minas Gerais, são evidentes os efeitos da ação dos ventos nos povoamentos, com a ocorrência e intensidade desses ventos afetando o desenvolvimento dos plantios e as características da madeira, interferindo significativamente em suas propriedades e no produto final. Melo (2004), trabalhando com intensidade de quebra de árvores por correntes de ventos em clones de Eucalyptus com 24 meses de idade, plantados em topografias diferentes, encontrou que clones que apresentaram maiores níveis de tensões de crescimento resultaram em maiores níveis de tolerância das árvores às tempestades.

Dentro desse contexto, este trabalho objetivou avaliar tensões de crescimento e sua correlação com outras propriedades em árvores de diferentes clones do híbrido Eucalyptus urophylla $\times$ Eucalyptus grandis. A coleta foi realizada nas áreas com intensidade de quebras das árvores em função dos ventos, através do método não destrutivo da medição da deformação residual longitudinal (DRL).

\section{MATERIAL E MÉTODOS}

\subsection{Procedência e amostragem do material}

No presente estudo foram utilizados dez clones do híbrido Eucalyptus urophylla $\times$ Eucalyptus grandis com 30 e 35 meses de idade, plantados em locais de baixada com altitude de $250 \mathrm{~m}$ em espaçamento de $3,33 \times 3,00 \mathrm{~m}$. A região de baixada foi escolhida por apresentar árvores mais vulneráveis à quebra pelo vento, devido à ausência dos ventos climatológicos.

O material é procedente de plantios florestais da Empresa Celulose Nipo-Brasileira S.A. (CENIBRA), localizada no município de Belo Oriente, na região do Vale do Rio Doce, a leste do Estado de Minas Gerais.

A região se encontra a $42^{\circ} 22^{\prime} 30^{\prime \prime}$ de longitude Sul e a $19^{\circ} 15^{\prime} 00^{\prime \prime}$ de latitude Oeste, com altitude, nos locais de plantio, de 230 a $500 \mathrm{~m}$. O clima, de acordo com Koppen, classifica-se em Aw - clima tropical, com 
inverno seco (entre os meses de maio e setembro) e estação chuvosa no verão, manifestando-se entre os meses de novembro e abril.

\subsection{Deformação residual longitudinal}

Foram destinadas à medição da deformação residual longitudinal (DRL), dez árvores por clones. Em seguida, três destas foram abatidas e coletaram-se três toras por árvore até a altura de $6 \mathrm{~m}$ do fuste comercial, cada uma com 2 m de comprimento.

As árvores foram selecionadas a partir do diâmetro médio na altura de 1,30 m de altura do solo, com os respectivos diâmetros iguais ou próximos da média entre as trinta árvores de uma parcela de $5 \times 6$ árvores. Optou-se por árvores eretas, com boas condições fitossanitárias, sem bifurcação e fora da área de bordadura.

A medição referente à DRL foi realizada nas árvores em pé, a 1,30 m de altura do solo nas diferentes direções: norte, sul, leste e oeste ou nordeste, sudoeste, noroeste e sudeste, de acordo com o sentido no qual houve maior predominância da queda de árvores após a ação dos ventos. A coleta da DRL em campo foi realizada no mês de janeiro.

As medições foram realizadas de acordo com o método não destrutivo do "CENTRE DE COOPERATION INTERNATIONALE EN RECHERCHE AGRONOMIQUE POUR LE DÉVELOPPEMENT-DÉPARTEMENT FORÊT” - CIRAD - Forêt ("Growth Strain Gauge" - Medidor de Deformação de Crescimento), que consiste em um relógio comparador digital - extensômetro, que tem como função informar a DRL por meio da compressão das fibras, uma vez que a tensão é liberada.

Ao extensômetro são conectados dois pinos, com uma distância entre eles de $45 \mathrm{~mm}$ na direção da grã, fixado no tronco da árvore sem a casca na altura de 1,30 $\mathrm{m}$ do solo. Posteriormente ao ajuste do aparelho, realizou-se um furo de $2 \mathrm{~cm}$ de diâmetro com um arco de pua, entre os pinos, a fim de liberar as tensões refletidas na contração longitudinal das fibras, obtendo assim a deformação residual longitudinal da madeira (Figura 1). Ao liberar esta tensão, o valor em milímetro é registrado diretamente no visor do relógio digital.

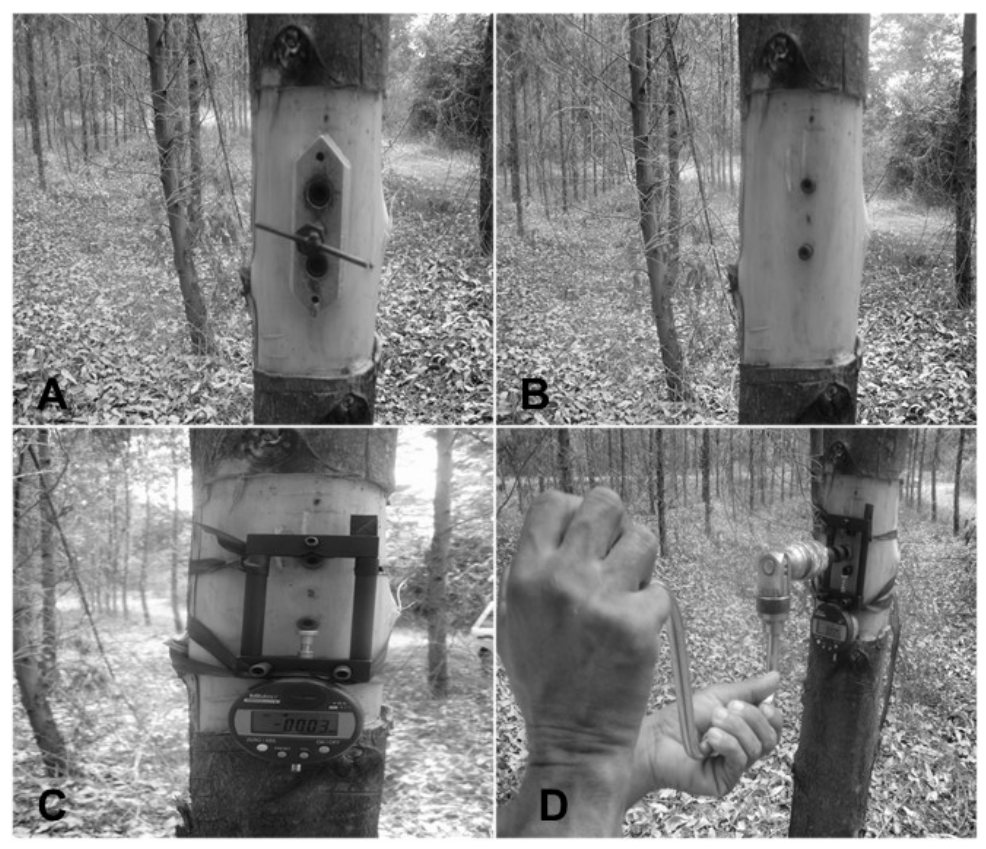

Figura 1. Medição da deformação residual longitudinal (DRL): (A) Fixação dos pinos a distância de $45 \mathrm{~mm}$ com o auxílio de um gabarito; (B) Pinos fixados ao tronco; (C) Ajuste do relógio digital apoiado nos pinos; (D) Uso do arco de pua para liberar as tensões.

Figure 1. Measuring of longitudinal residual strain (DRL): (A) Fixation of the pins at distance of $45 \mathrm{~mm}$ with the aid of a template; (B) Pins fixed the stem; (C) Adjust of the digital clock supported in the pins; (D) Use of bow drill to release tension. 
Os valores da DRL foram tomados na ausência de vento, uma vez que os valores na presença deste poderiam ser superestimados, pois com o movimento das árvores, as forças de sustentação interna poderiam oscilar, influenciando assim no resultado.

A partir dos valores obtidos da deformação residual longitudinal, juntamente com o módulo de elasticidade encontrado, foram determinadas as tensões de crescimento das árvores, pelo emprego da seguinte fórmula (Equação 1).

$T C L=\frac{M O E \times D R L}{45}$

em que:

$\mathrm{TCL}=$ Tensão de crescimento longitudinal $\left(\mathrm{Kgf} / \mathrm{cm}^{2}\right)$; MOE $=$ Módulo de elasticidade $\left(\mathrm{Kgf} / \mathrm{cm}^{2}\right)$; $\mathrm{DRL}=$ Deformação residual longitudinal $(\mathrm{mm})$; 45 = Distância entre os dois pinos $(\mathrm{mm})$.

\subsection{Características da madeira}

As mensurações das dimensões da árvore, diâmetro na altura do peito (DAP) a 1,30 m do solo e altura total foram realizadas em campo com o uso de uma fita métrica. Os estudos da propriedade física (densidade básica) e mecânica (flexão estática) da madeira foram realizados no Laboratório de Ciências da Madeira, do Departamento de Ciências Florestais e da Madeira - DCFM da Universidade Federal do Espírito Santo - UFES.

A determinação da densidade básica e flexão estática da madeira foram realizadas com base no Método Brasileiro - MB -26 da Associação Brasileira de Normas Técnicas (ABNT, 1940). O ensaio mecânico foi executado com a madeira nas condições de verde, uma vez que as árvores, quando quebradas em campo pela ação dos ventos, se encontravam nas mesmas condições.

\subsection{Análises dos resultados}

Os dados foram dispostos e analisados, sendo encontrados os valores de média, desvio-padrão e coeficiente de variação. Para avaliar o grau de significância dos parâmetros avaliados entre os clones, bem como suas variações, foi realizada uma análise de variância e, quando significativa, aplicou-se o teste
Tukey a 5\% de probabilidade para a comparação de médias. Posteriormente, foi conduzido um estudo das correlações entre a deformação residual longitudinal e as características da árvore por meio da análise de Correlação de Pearson ao nível de significância de 5\%.

\section{RESULTADOS E DISCUSSÃO}

\subsection{Deformação residual longitudinal}

Verifica-se na Figura 2 a distribuição da DRL (mm) em torno do tronco, bem como os valores médios, desvio-padrão e coeficiente de variação para os dez clones do híbrido de Eucalyptus urophylla $\times$ Eucalyptus gradis .

A DRL decorrente das tensões de crescimento foi em média geral de 0,086 mm. Estatisticamente, o clone CNB001 apresentou o maior valor para a DRL, sendo de 0,116 mm, e os clones CNB005 e CNB015 o menor valor de $0,060 \mathrm{~mm}$. Os coeficientes de variação para o DRL entre os dez clones variaram de 34,14 a 129,87\%, demonstrando uma alta dispersão, podendo considerar o material heterogêneo.

As diferenças de leitura da DRL podem estar associadas a diversos fatores ambientais, em específico, o efeito da ação dos ventos, uma vez que os materiais de estudo estão plantados em regiões que, com a ocorrência e intensidade desses ventos, há até mesmo a quebras das árvores e afetam o desenvolvimento dos plantios e, consequentemente, as características da madeira.

Melo (2004), ao estudar clones de Eucalyptus aos dois anos de idade cultivados em áreas sujeitas a tempestade, encontrou valores médios para a DRL vaiando de 0,095 a 0,106 mm. Cardoso et al. (2005) encontraram um valor médio para a DRL de $0,065 \mathrm{~mm}$ para um clone de Eucalyptus com diferentes idades. Souza (2002) encontrou, em clone de híbridos naturais de Eucalyptus com idade de seis anos, valor médio de DRL de 0,090 mm. Os resultados obtidos no presente estudo, apesar de a procedência e o tipo do material genético serem diferentes dos outros estudos e também com idade bem inferior, foram semelhantes aos encontrados na literatura.

Os clones CNB007, CNB008, CNB012, CNB017 e CNB1206 apresentaram uma melhor distribuição da deformação em torno do tronco, já os demais clones apresentaram distribuições irregulares, o que pode 


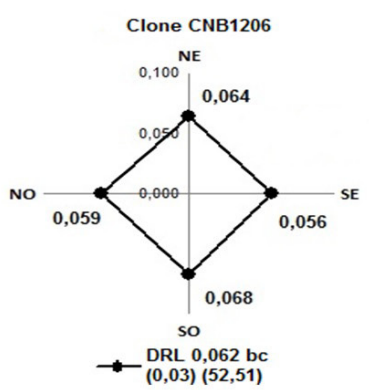

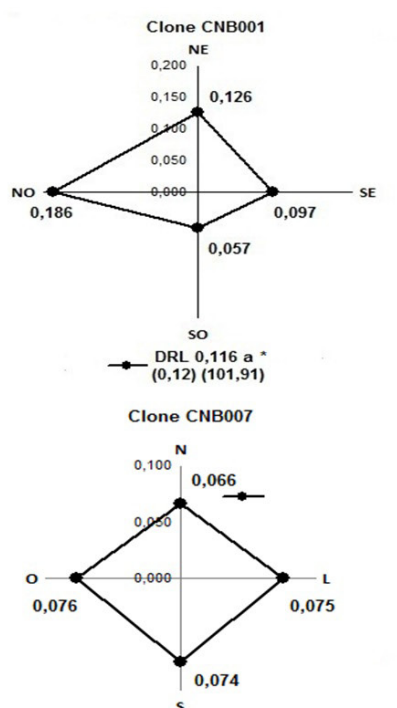
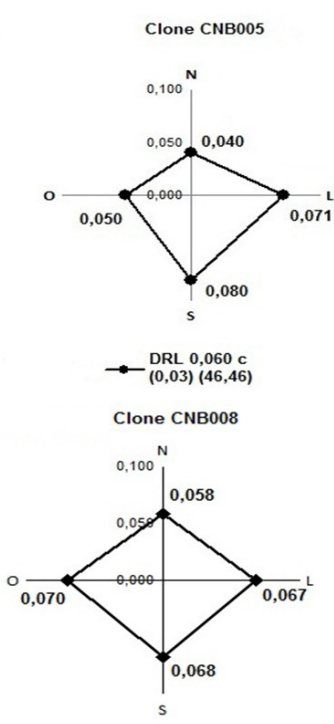

$S$
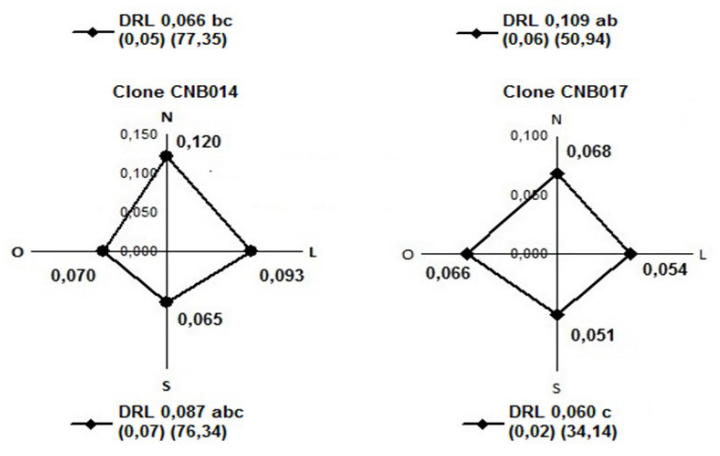

DRL 0,098 abc

$(0,13)(129,87)$

Clone CNB010

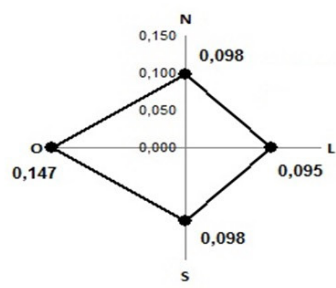

DRL 0,109 ab

$(0,06)(50,94)$

$(0,07)(76,34)$
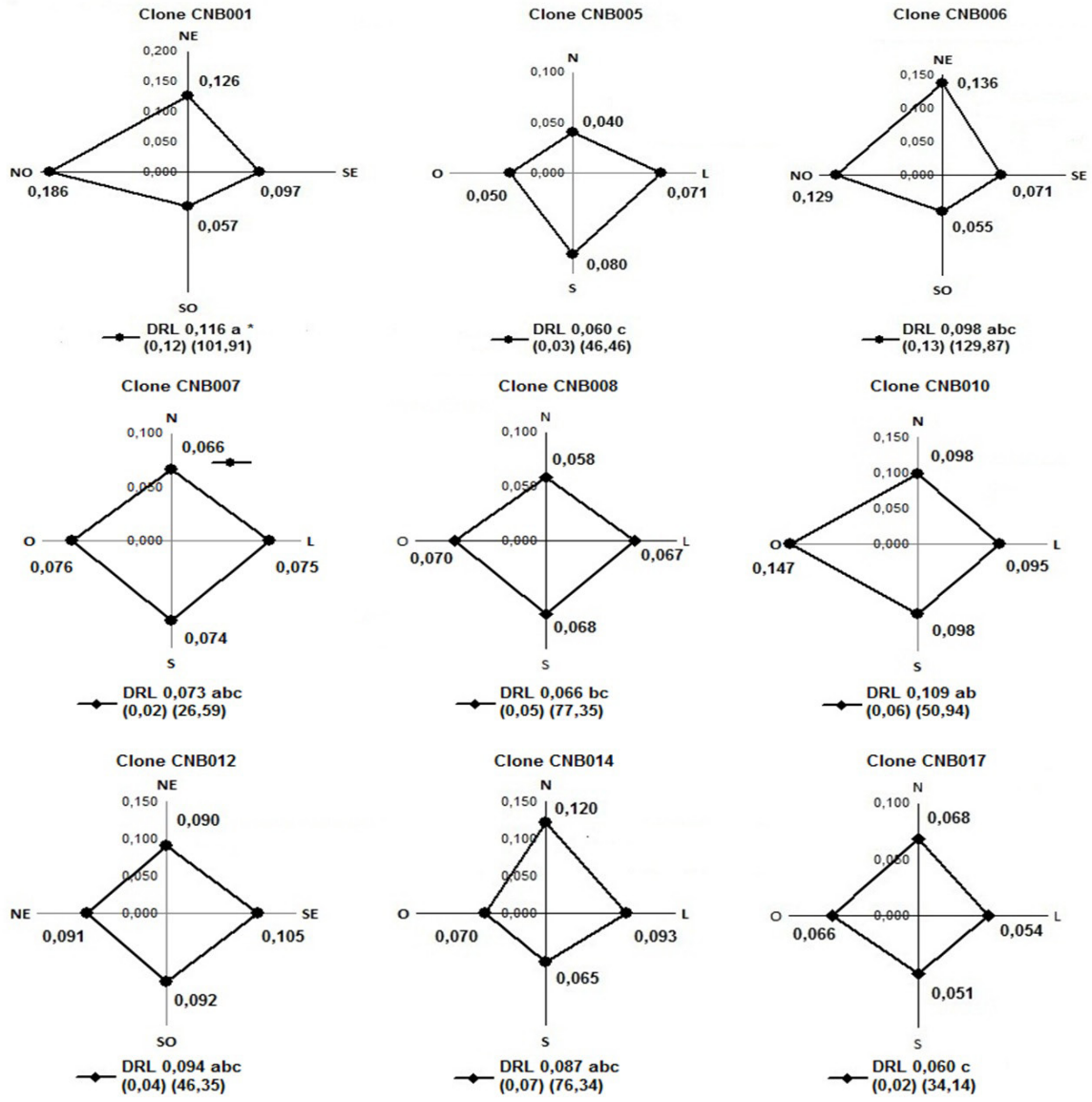

$(0,04)(46,35)$

Figura 2. Representação gráfica dos valores médios $(\mathrm{mm})$, desvio-padrão $(\mathrm{mm})$ e coeficiente de variação (\%) das deformações longitudinais residuais nas diferentes orientações no tronco para os dez clones do híbrido Eucalyptus urophylla $\times$ Eucalyptus grandis. ${ }^{\star}$ Valores médios seguidos de mesma letra não diferem estatisticamente entre si (Tukey, $\mathrm{p}>0,05$ ).

Figure 2. Graphical representation of mean values $(\mathrm{mm})$, standard deviation $(\mathrm{mm})$ and coefficient of variation $(\%)$ of the longitudinal residual strains in different orientations in the stem to the hybrid Eucalyptus urophylla $\times$ Eucalyptus grandis. ${ }^{\star}$ Mean values followed by the same letter do not statistically different from each other (Tukey, $\mathrm{p}>0.05$ ).

resultar na formação de madeira de tração. A variação entre os clones pode ser justificada pela ação dos ventos, visto que a intensidade da incidência pode mudar em função da direção e sentido dos ventos e do local (altitude).
Os valores da tensão de crescimento longitudinal (Mpa) para os dez clones do híbrido Eucalyptus urophylla $\times$ Eucalyptus grandis estão apresentados na Figura 3 .

Em relação à TCL, o valor médio entre os clones foi de 12Mpa, o clone CNB001 foi o que apresentou o 


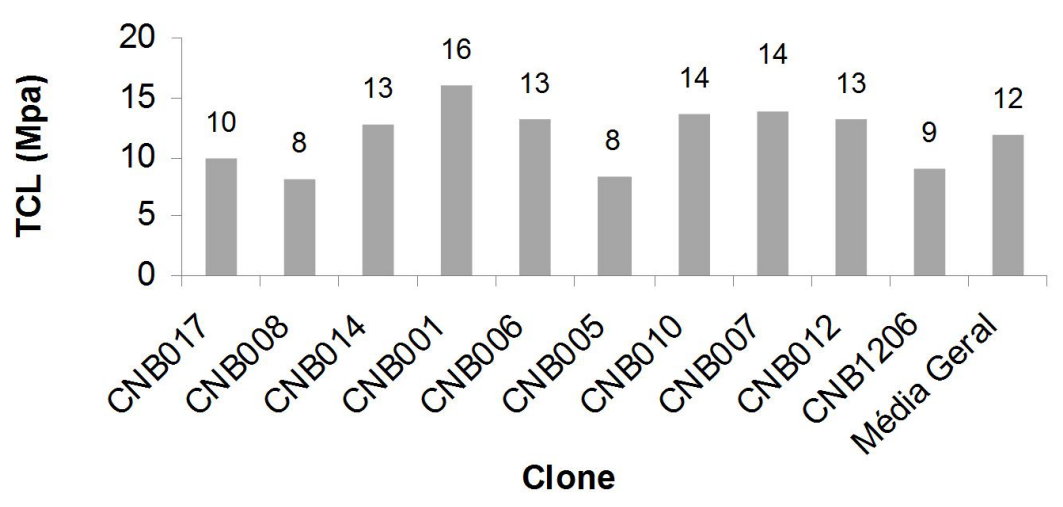

Figura 3. Representação gráfica dos valores médios da tensão de crescimento longitudinal (Mpa) para os dez clones do híbrido Eucalyptus urophylla $\times$ Eucalyptus grandis.

Figure 3. Graphical representation of mean values of longitudinal growth stress (Mpa) to the hybrid Eucalyptus urophylla $\times$ Eucalyptus grandis clones.

maior valor para a TCL, sendo 16Mpa. Melo (2004), ao avaliar quatro clones de Eucalyptus com idade de 24 meses, obteve valores de tensão de crescimento longitudinal variando de 11,18 a 14,51Mpa, concluindo que os maiores níveis de tensões de crescimento das árvores conduzem a maiores níveis de tolerância destas a tempestades. Nos resultados obtidos por Braz et al. (2014) ao classificar os mesmos clones quanto à resistência simulando a ação dos ventos, verificou-se que não poderia afirmar que os clones que apresentassem maiores tensões de crescimento, consequentemente apresentariam maior tolerância quando submetidos a ventos. Trugilho (2005) encontrou valores variando entre 13,63 e 43,93Mpa para diferentes clones de Eucalyptus com seis anos de idade. Os valores para a tensão de crescimento foram baixos, semelhantes aos citados na literatura para idades menores.

\subsection{Correlações entre a DRL e outras características da madeira}

Na Tabela 1 seguem os valores médios das variáveis de crescimento da árvore, a densidade básica da madeira e os valores do módulo de elasticidade (MOE), módulo de ruptura (MOR) e trabalho absorvido obtido através do ensaio mecânico de flexão estática da madeira para os dez clones do híbrido Eucalyptus urophylla $\times$ Eucalyptus gradis, os quais foram correlacionados com a valores da DRL.
$\mathrm{O}$ diâmetro à altura do peito (DAP) médio foi de $12,34 \mathrm{~cm}$, sendo que os clones CNB001 e o CNB005 apresentaram respectivamente o menor e o maior DAP $(10,32$ e 13,34 cm). A altura total (HT) média foi de $17,82 \mathrm{~m}$, a maior e menor HT ocorreu para o clone CNB001 (14,89m) e clone CNB012 (20,10 m).

O valor médio para a densidade básica da madeira foi $0,395 \mathrm{~g} / \mathrm{cm}^{3}$, o clone CNB014 e CNB017 apresentaram o maior valor, ambos com $0,429 \mathrm{~g} / \mathrm{cm}^{3}$, e o clone CNB007 o menor com $0,339 \mathrm{~g} / \mathrm{cm}^{3}$. Os valores médios para o MOE, MOR e o trabalho absorvido foram de $60965 \mathrm{Mpa}, 544 \mathrm{Mpa}$ e 10J, respectivamente. O clone CNB017 foi o que apresentou o maior MOE e MOR (7138 e 64Mpa), e o clone CNB1226 o maior trabalho absorvido (13J).

A Tabela 2 apresenta os coeficientes de correlação encontrados entre a deformação residual longitudinal em função das dimensões das árvores e das propriedades física e mecânica da madeira para os dez clones do híbrido Eucalyptus urophylla $\times$ Eucalyptus grandis.

As deformações residuais longitudinais não apresentaram coeficiente de Correlação de Pearson significativo ao nível de $5 \%$ com as variáveis de crescimento das árvores e com as propriedades da madeira analisadas para cada material genético isoladamente, indicando uma fraca influência dessas características sobre este parâmetro para os clones do híbrido Eucalyptus urophylla $\times$ Eucalyptus grandis. 
Tabela 1. Valores médios das características das árvores e da madeira para os dez clones do híbrido Eucalyptus urophylla $\times$ Eucalyptus grandis.

Table 1. Average values of the characteristics of trees to the hybrid Eucalyptus urophylla $\times$ Eucalyptus grandis clones.

\begin{tabular}{|c|c|c|c|c|c|c|c|}
\hline \multirow[b]{2}{*}{ Clone } & \multicolumn{7}{|c|}{ Características $^{1}$} \\
\hline & $\begin{array}{c}\text { DRL } \\
(\mathbf{m m})\end{array}$ & $\begin{array}{l}\text { DAP } \\
(\mathrm{cm})\end{array}$ & $\begin{array}{l}\text { HT } \\
(\mathrm{m})\end{array}$ & $\begin{array}{c}\mathrm{DB} \\
\left(\mathrm{g} / \mathrm{cm}^{2}\right)\end{array}$ & $\begin{array}{l}\text { MOE } \\
\text { (Mpa) }\end{array}$ & $\begin{array}{l}\text { MOR } \\
\text { (Mpa) }\end{array}$ & $\begin{array}{c}\text { TAB } \\
\text { (J) }\end{array}$ \\
\hline CNB001 & 0,116 & 10,82 & 14,89 & 0,382 & 6051 & 56 & 11 \\
\hline CNB005 & 0,060 & 13,34 & 19,24 & 0,397 & 6094 & 56 & 7 \\
\hline CNB006 & 0,098 & 12,35 & 17,31 & 0,396 & 5979 & 53 & 11 \\
\hline CNB007 & 0,073 & 11,81 & 17,08 & 0,338 & 5554 & 50 & 10 \\
\hline CNB008 & 0,066 & 12,13 & 15,78 & 0,394 & 5514 & 49 & 10 \\
\hline CNB010 & 0,109 & 13,08 & 19,66 & 0,369 & 5544 & 47 & 11 \\
\hline CNB012 & 0,094 & 12,80 & 20,10 & 0,423 & 6173 & 52 & 7 \\
\hline CNB014 & 0,087 & 11,78 & 16,68 & 0,429 & 6506 & 60 & 8 \\
\hline CNB017 & 0,060 & 12,45 & 17,48 & 0,429 & 7138 & 64 & 9 \\
\hline CNB1206 & 0,062 & 12,80 & 19,93 & 0,396 & 6412 & 57 & 13 \\
\hline Média & 0,086 & 12,34 & 17,82 & 0,395 & 6096 & 54 & 10 \\
\hline
\end{tabular}

${ }^{1} \mathrm{DRL}=$ deformação residual longitudinal; $\mathrm{DAP}=$ diâmetro a $1,30 \mathrm{~m}$ de altura do solo; $\mathrm{HT}=$ altura total da árvore; $\mathrm{DB}=$ densidade básica; $\mathrm{MOE}=$ módulo de elasticidade a flexão estática; $\mathrm{MOR}$ = módulo de ruptura a flexão estática; $\mathrm{TAB}$ = trabalho absorvido.

Tabela 2. Correlações entre a DRLs e outras características da madeira para os dez clones do híbrido Eucalyptus urophylla $\times$ Eucalyptus grandis.

Table 2. Correlations between DRLs and other wood characteristics to the hybrid Eucalyptus urophylla $\times$ Eucalyptus grandis clones.

\begin{tabular}{lcccccc|}
\hline & \multicolumn{7}{c|}{ Características $^{1}$} \\
\cline { 2 - 7 } Clone & DAP & HT & DB & MOE & MOR & TAB \\
\hline CNB001 & 0,577 & 0,243 & 0,243 & 0,243 & $-0,418$ & $-0,018$ \\
\hline CNB005 & 0,211 & $-0,176$ & $-0,176$ & $-0,176$ & 0,176 & 0,162 \\
\hline CNB006 & $-0,619$ & $-0,277$ & $-0,277$ & $-0,277$ & 0,076 & $-0,255$ \\
\hline CNB007 & $-0,230$ & $-0,230$ & $-0,230$ & $-0,230$ & $-0,075$ & $-0,346$ \\
\hline CNB008 & $-0,254$ & 0,343 & 0,343 & 0,343 & 0,650 & 0,348 \\
CNB010 & 0,286 & $-0,638$ & $-0,638$ & $-0,638$ & 0,623 & 0,296 \\
\hline CNB012 & 0,183 & $-0,559$ & $-0,559$ & $-0,559$ & 0,304 & $-0,544$ \\
\hline CNB014 & $-0,124$ & 0,052 & 0,052 & 0,052 & $-0,808$ & $-0,608$ \\
\hline CNB017 & 0,699 & 0,072 & 0,072 & 0,072 & $-0,196$ & $-0,027$ \\
\hline CNB1206 & $-0,231$ & $-0,768$ & $-0,768$ & $-0,768$ & $-0,874$ & 0,356 \\
\hline
\end{tabular}

${ }^{1} \mathrm{DAP}=$ diâmetro a $1,30 \mathrm{~m}$ de altura do solo; $\mathrm{HT}$ = altura total da árvore; $\mathrm{DB}=$ densidade básica; $\mathrm{MOE}$ = módulo de elasticidade a flexão estática; $\mathrm{MOR}$ = módulo de ruptura a flexão estática; $\mathrm{TAB}=$ trabalho absorvido.

Muneri et al. (2000) obtiveram correlações positivas e significativas da DRL com a altura e diâmetro das árvores de Eucalyptus cloeziana. Já Souza et al. (2003), ao estudarem clones de híbridos naturais do gênero Eucalyptus, com seis anos de idade, concluíram que a DRL não apresentou correlação com as características de dendrométricas (DAP e a altura total das árvores).

Isso demonstra o quanto são diversificadas as interações das DRLs com as características de crescimento entre as espécies do gênero Eucalyptus, apresentando-se dinâmicas. Clair et al. (2003) também encontraram relações significativas entre as tensões de crescimento e as propriedades físicas e mecânicas da madeira. Lima et al. (2004), ao estudarem as correlações da DRL com outras propriedades da madeira para híbrido natural de Eucalyptus grandis W. Hill. Ex Maiden e Eucalyptus grandis W. Hill. Ex Maiden $\times$ Eucalyptus urophylla S.T. Blake, concluíram que entre as várias propriedades da madeira, apenas a densidade básica apresentou correlação significativa e positivamente 
com a DRL, quando todos os materiais genéticos foram analisados conjuntamente. Ainda os mesmos autores relataram que as tensões de crescimento são mais fortemente correlacionadas com as características de crescimento das árvores do que com as propriedades mecânicas da madeira.

De acordo com Rodrigues et al. (2008), a diferença da DRL nos Eucalyptus pode estar associada às variações na madeira, em função da formação da madeira de tração.

\section{CONCLUSÕES}

Os elevados valores para coeficiente de determinação mostraram uma heterogeneidade entre os indivíduos analisados, o que pode ser utilizado em programas de melhoramento florestal para a seleção de material superior com características desejáveis.

As características de crescimento das árvores, a densidade básica, o MOE e MOR e trabalho absorvido não foram suficientes para explicar o comportamento da deformação residual longitudinal.

\section{AGRADECIMENTOS}

Os autores agradecem à Empresa de Celulose Nipo Brasileira S.A. (CENIBRA), pela disponibilidade do material de estudo e pelo apoio logístico de trabalho. Agradecem também ao CNPQ pela concessão de bolsa de produtividade em pesquisa a um dos autores

\section{STATUS DA SUBMISSÃO}

Recebido: 15 jan., 2013

Aceito: 7 set., 2016

\section{AUTOR(ES) PARA CORRESPONDÊNCIA}

\section{Rafael Leite Braz}

Departamento de Ciência Florestal, Universidade Federal Rural de Pernambuco - UFRPE, Rua Dom Manoel de Medeiros, s/n, Dois Irmãos, CEP 52171-900, Recife, PE, Brasil e-mail: rafaellbraz@yahoo.com.br

\section{REFERENNCIAS}

Associação Brasileira de Normas Técnicas - ABNT. MB-26/40: ensaios físicos e mecânicos da madeira. Rio de Janeiro; 1940. 16 p.

Baena ES. A utilização de Eucalyptus saligna Smith e Eucalyptus grandis Hill jovens na confecção de sarrafos [dissertação]. Piracicaba: Escola Superior de Agricultura de Luíz de Queiroz, Universidade de São Paulo; 1982.

Braz RL, Oliveira JTS, Rosado AM, Vidaurre GB, Paes JB. Parâmetros dendrométricos e resistência mecânica das árvores de clones de Eucalyptus em áreas sujeitas à ação dos ventos. Ciência Florestal 2014; 24(4): 947-958. http://dx.doi.org/10.5902/1980509816608.

Cardoso AA Jr, Trugilho PF, Lima JT, Rosado SCS, Mendes LM. Deformação residual longitudinal em diferentes espaçamentos e idade em clones de híbridos de Eucalyptus. Cerne 2005; 11(3): 218-224.

Carvalho AM, Gonçalves MPM, Amparado KF, Latorraca JVF, Garcia RA. Correlações da altura e diâmetro com tensões de crescimento em árvores de Corymbia citriodora e Eucalyptus urophylla. Árvore 2010; 34(2): 323-331. http:// dx.doi.org/10.1590/S0100-67622010000200015.

Clair B, Ruelle J, Thibaut B. Relationship between growth stresses, mechanic physical properties and proportion of fibre with gelatinous layer in Chestnut (Castanea sativa Mill.). Holzforschung 2003; 57(2): 189-195. http://dx.doi. org/10.1515/HF.2003.028.

Del Menezzi CH. Utilização de um método combinado de desdobro e secagem para a produção de madeira serrada de Eucalyptus grandis W. Hill ex-Maiden e E.cloesiana F. Muell [dissertação]. Piracicaba: Escola Superior de Agricultura Luiz de Queiroz, Universidade de São Paulo; 1999.

Dinwoodie JM. Growth stresses in timber: a review of literature. Forestry 1966; 39(2): 162-170. http://dx.doi. org/10.1093/forestry/39.2.162.

Hillis WE, Brown AG. Eucalyptus for wood production. Melbourne: CSIRO; 1978.

Huang YS, Hung LF, Kuo-Huang LL. Biomechanical modeling of gravitropic response of branches: roles os asymmetric periohery growth strain versus self-weight bending effect. Trees 2010; 24(6): 1151-1161. http://dx.doi. org/10.1007/s00468-010-0491-0.

Kubler H. Growth stresses in trees and related wood properties. Forest Products Abstracts 1987; 10(3): 61-119.

Latorraca JVF, Albuquerque CEC. Efeito do rápido crescimento sobre as propriedades da madeira. Floresta e Ambiente 2000; 7(1): 279-291.

Lima JT, Trugilho PF, Rosado SCZ, Cruz CR. Deformações residuais longitudinais decorrentes de tenções de crescimento em Eucalyptus e sua associação com outras propriedades. Árvore 2004; 28(1): 107-116. 
Melo VM. Variação nas produtividades da madeira de clones de Eucalyptus cultivados em diferentes topografiase sujeitas a tempestade [dissertação]. Lavras: Universidade Federal de Lavras; 2004.

Muneri A, Knigth J, Legate W, Palmer G. Relationships between surface longitudinal growth strain and tree size, wood properties and timber distorcion of 4 years old plantation grown Eucalyptus cloeziana. In: IUFRO Conference: the future of eucalyptus for wood products; 2000; Lauceston. Lauceston: IUFRO; 2000. p. 292-300.

Opie JE, Curtin RA, Incoll WD. Stand management. In: Hillis WE, Brown AG, editores. Eucalypts for wood production. Sydney: CSIRO; 1984.

Rech C, Silva JC. Melhoramento genético para a qualidade da madeira. Madeira 2001a; 48-54. Edição especial.

Rech C, Silva JC. Características intrínsecas da madeira. Madeira 2001b; 60-65. Edição Especial.

Rodrigues EAC, Rosado SCS, Trugilho PF, Santos AM. Seleção de clones de Eucalyptus para as propriedades físicas da madeira avaliadas em árvores no campo. Cerne 2008; 14(2): 147-152.
Souza MAM, Trugilho PF, Lima JT, Rosado SCS. Deformação residual longitudinal e sua relação com algumas características de crescimento e da madeira em clones de Eucalyptus. Floresta 2003; 33(3): 275-284. http:// dx.doi.org/10.5380/rf.v33i3.2261.

Souza MAM. Deformação residual longitudinal (DRL) causada pelas tensões de crescimento em clones de híbridos de Eucalyptus [dissertação]. Lavras: Universidade Federal de Lavras; 2002.

Trugilho PF, Lima JT, Pádua FA, Soragi LC, Andrade CR. Deformação residual longitudinal (DRL) e tangencial (DRT) em seis clones de Eucalyptus spp. Cerne 2006; 12(3): 279-286.

Trugilho PF. Tensão de crescimento em árvores vivas de clones de Eucalyptus spp. e de Eucalyptus dunnii maiden e propriedades da sua madeira [tese]. Curitiba: Universidade Federal do Paraná; 2005.

Wyk JL Van. Hardwood sawmilling can have a brtight future in South Africa. Southern African Forestry Journal 1978; 109(1): 47-53. http://dx.doi.org/10.1080/2070262 0.1978 .10433505 . 